\title{
Zu Risiken und Nebenwirkungen fragen Sie Ihren Arzt oder Apotheker ...
}

Markus Gnädinger

Institut für Hausarztmedizin und Versorgungsforschung der Universität Zürich
Korrespondenz:

Dr. med. Markus Gnädinger

Facharzt für Innere Medizin

Birkenweg 8

CH-9323 Steinach

markus.gnaedinger[at]hin.ch
Fiktive Erzählungen können unterhaltsam sein, doch die selbsterlebten Geschichten sind mindestens so spannend - zumindest für den Erzähler.

Im Jahr 2002 präsentierte ein Chefarzt der Medizin an der monatlichen Hausärzte-Fortbildung den Fall einer Patientin mit Parkinson-Krankheit (PD) und schweren osteoporotischen Wirbelkörperfrakturen der Lendenwirbelsäule. Und wie es der Zufall wollte, brach sich kurz danach eine meiner eigenen Patientinnen bei einem Sturz im Altersturnen ebenfalls den Lendenwirbel. Auch diese Patientin hatte eine schon lange bestehende PD. Ob diesen parallelen Fällen überlegte ich mir, ob die PD und die Osteoporose eine gemeinsame Schnittstelle haben könnten, und begann mit einer Literatursuche. Es zeigte sich nach dem damaligen Wissenstand, dass PD-Patienten gehäuft eine verminderte Knochendichte (BMD) bis hin zur Osteoporose (T-Wert $<2,5$ ) und Frakturen hatten. Zudem fanden sich häufig erniedrigte Vitamin-D-Werte. Es gab aber auch Hinweise für eine knochenschädigende Wirkung der PD-Medikamente. Ich meldete den Fall meiner Patientin als vermutete Medikamenten-Nebenwirkung. Der Bescheid lautete, PD-Patienten seien immobil (so viel zum Altersturnen!), und bei postmenopausalen Frauen komme Osteoporose halt vor.

Ich meldete später auch einen Fall von Osteopenie bei einem Mann, der wegen seiner unruhigen Beine (RLS) L-Dopa einnehmen musste. Auch hier: «Kommt halt so vor!»

Ich wollte eine hausärztliche Primärstudie bei neudiagnostizierten PD-Patienten lancieren und kontrollieren, ob nach L-Dopa-Therapie die BMD abund die Urin-Crosslinks zunähmen. Diese Studie scheiterte an der Finanzierung und an den komple-

\section{Checkliste}

- Betreuen Sie Patienten mit Parkinson-Erkrankung?

- Sind Sie sich des Risikos dieser Patienten für Osteoporose und Frakturen bewusst?

- Sind bei allen Ihren Parkinsonpatienten die Empfehlungen des Institute of Medicine (IOM) erfüllt, wonach Senioren über 70 täglich $800 \mathrm{E}$ Vitamin D und eine Gesamtmenge von mindestens 1200 mg Kalzium zuführen sollten? [2]

- Verschreiben Sie L-Dopa-haltige Präparate (insb. Madopar ${ }^{\oplus}$, Sinemet $^{\oplus}$ )?

\section{Ceci est un médicament. Deman- dez conseil à votre spécialiste et lisez la notice d'emballage}

Les patients atteints de Parkinson risquent de tomber et de se casser quelque chose. En effet, en dehors des troubles neurologiques, une densité osseuse amoindrie favorise les fractures. De plus, il est possible que les médicaments contenant du L-Dopa soient impliqués dans le développement de l'ostéoporose.

xen Formalitäten (Multicenterstudie, Ethik, Versicherung usw.).

Nun liess ich die Sache ruhen. Erst im Jahr 2010 nahm ich wieder einen Anlauf und konnte mit zwei Koautoren und durch Hilfe eines Stipendiums der Akademie der Medizinischen Wissenschaften einen Übersichtsartikel über die Problematik erstellen. Diese Arbeit ist $2011 \mathrm{im}$ Swiss Medical Weekly erschienen [1]. Sie bestätigte, dass ein Zusammenhang zwischen der PD und Knochenproblemen vorhanden ist. Als Ursache des wahrscheinlich multifaktoriellen Problems kommen in Frage: langjähriger Vitamin-D-Mangel, Immobilität, Mangelernährung und L-Dopa-haltige PD-Medikamente.

Mit Hinweis auf diese Übersichtsarbeit liess ich meine beiden oben erwähnten Nebenwirkungsmeldungen nochmals im Lichte der aktuellen Literatur bewerten. Die Beurteilung hatte sich nun von «unwahrscheinlich» zu «möglich» geändert.

Ein Briefwechsel mit Swissmedic folgte. Ich beanstandete dabei die fehlende Patienteninformation über mögliche schädliche Auswirkungen von LDopa plus Benserazid (Madopar ${ }^{\circledR}$ ) oder Carbidopa $\left(\right.$ Sinemet $\left.{ }^{\oplus}\right)$. Meine Argumentation war die folgende: L-Dopa selber stört die Kollagen-Biosynthese und damit den Aufbau eines gesunden Knochens. Zudem führt die Therapie mit L-Dopa plus Decarboxylasehemmer zu einer massiven Hyperhomozysteinämie (in meiner Praxis ca. 2,5-mal die obere Norm). Hohe Homozysteinspiegel schaden (unter anderem) dem Knochen. Die Gabe von Vitamin B12 und Folsäure kann die Hyperhomozysteinämie und die Zeichen eines raschen Knochenabbaus (Pryridinolin-Crosslinks im Urin) reduzieren. Auch die Erweiterung des 


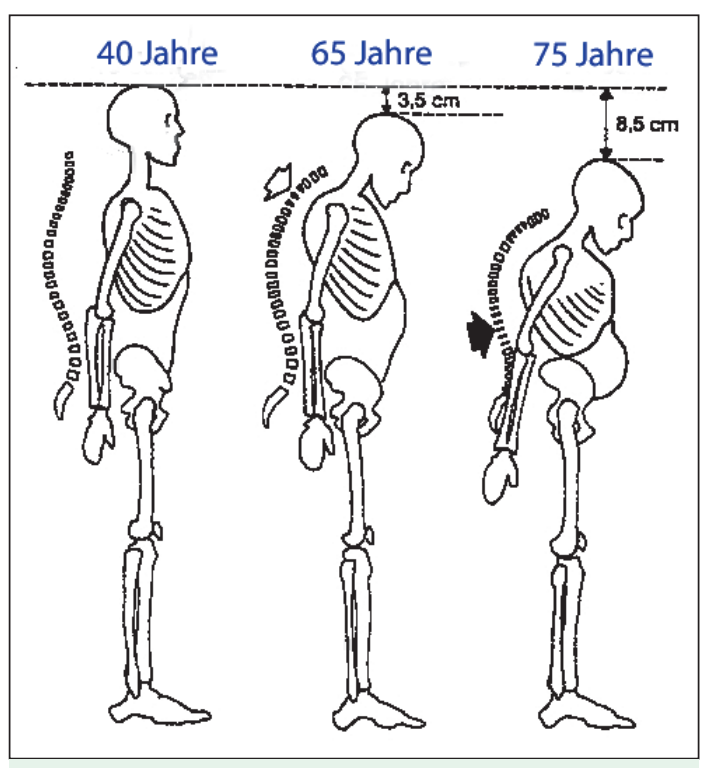

Parkinson-Patienten leiden gehäuft unter verminderter Knochendichte bis hin zur Osteoporose. Ist dies auch Folge einer knochenschädigenden Wirkung von Parkinson-Medikamenten?

Therapieschemas durch einen Hemmer der Carboxy-Methyl-Transferase (COMT) mit Tolcapon (Tasmar $^{\oplus}$ ) oder Entacapon (mono: Comtan ${ }^{\circledast}$, combi: Stalevo $^{\circledast}$ ) kann einer Hyperhomozysteinämie entgegenwirken.

Die Antwort von Swissmedic lautete (nur Ausschnitte zitiert): «Es gibt keine Daten, die mit einem geeigneten Studiendesign die unabhängige Auswirkung der Antiparkinson-Medikation auf den Knochen und die Auslösung bzw. Verschlechterung oder Beschleunigung einer Osteoporose belegen (...) im Gegenteil, aus den Daten der klinischen Studien geht hervor, dass die Grundkrankheit Parkinson selbst als schwerwiegendes Risiko für die Osteoporose angesehen werden muss, wobei die Medikation, wenn überhaupt, nur eine untergeordnete Rolle spielen sollte. Das Spontanmeldesystem in der Schweiz und weltweit hat bisher auch noch kein Signal in Bezug auf durch die Anti-Parkinson-Medikation induzierte Knochenschäden generiert. Zudem wird bereits gemahnt Patienten (...) mit Osteomalazie sollten (...) ärztlich besonders beobachtet werden'.»

Ist diese Beurteilung unserer MedikamentenBehörde konklusiv? Die PD-Patienten sind ja weder die jüngsten noch die gesündesten, so dass kaum ein Arzt auf die Idee kommen dürfte, dass die Fraktur seines Patienten etwas mit den PD-Medikamenten zu tun haben könnte, die der Patient zu diesem Zeitpunkt schon jahrelang einnimmt. Leider wäre die Meldepsychologie aber auch so, wenn es einen tatsächlichen Zusammenhang gäbe, so dass dem Fehlen von Spontanmeldungen keinerlei Beweiskraft innewohnt.

In Beipackzetteln wird doch alles Mögliche genannt: Kopfweh, Schwindel, Müdigkeit und Schlaflosigkeit sind praktisch bei jedem Präparat erwähnt und müssten wohl sogar bei Traubenzuckerpillen angewarnt werden. Es käme doch niemandem in den Sinn, einem Erwachsenen einzubläuen, vor dem Überqueren einer Strasse zu schauen und zu horchen, ob sich kein Fahrzeug nähert! Darf eine Behörde wie Swissmedic wirklich nur warnen, wenn ein Sachverhalt klar und erwiesen ist? Wäre es vertretbar, nach einem Seebeben nur eine TsunamiWarnung zu erlassen, wenn felsenfest erwiesen ist, dass eine gefährliche Welle bereits heranrollt?

Warnungen braucht es, wenn mögliche, vor allem nicht allgemein bekannte und vermeidbare Gefahren lauern. Wenn Sie selber morgen Madopar ${ }^{\circledR}$ oder Sinemet ${ }^{\circledR}$ schlucken müssten, möchten Sie doch auch nach der Maxime dokumentiert sein: «Was drin ist, muss auch drauf stehen.» Immerhin hat Swissmedic die folgende Forderung aus unserem Review ausdrücklich bestätigt: «Für jedes neu zur Langzeittherapie zugelassene Medikament muss dessen Sicherheit hinsichtlich des Knochenstoffwechsels dokumentiert werden.» Diese für mich überraschende Zustimmung hat mich sehr gefreut und macht für die Zukunft optimistisch.

Im Anschluss an den frustrierenden Briefwechsel mit Swissmedic schrieb ich die Herstellerfirmen von Madopar $^{\circledast}$ und Sinemet ${ }^{\circledast}$ an. Für deren ausstehende Antworten wurden mehrere Monate veranschlagt. Im provisorischen Bescheid wurden ebenfalls die fehlenden Spontanmeldungen moniert. Seit meiner Intervention bei der klinischen Pharmakologie über Swissmedic zu den Herstellerfirmen ist nun mehr als ein halbes Jahr vergangen - sollte ich zuwarten bis zur definitiven Absage der Hersteller? Nein, somit entschied ich mich für den Gang in die Öffentlichkeit und zum Verfassen dieses Textes.

Was können Sie tun, liebe Kolleginnen und Kollegen? Wie in der Antwort von Swissmedic erwähnt, «glauben» die Behörden erst an eine deklarationspflichtige Nebenwirkung eines bestimmten Medikaments, wenn kontrollierte Studien diese aufgezeigt haben oder eine signifikante Zahl von Spontanmeldungen erfolgt ist. Kontrollierte Studien mit L-Dopahaltigen Medikamenten sind aus ethischen Gründen heute nicht mehr möglich. Es bleiben die Nebenwirkungs-Spontanmeldungen.

Hiermit möchte ich Sie deshalb auffordern, alle Ihre PD-Patienten mit Osteoporose mit einem gelben Formular (UAW) zu melden (am besten schicken Sie die Formulare an die Klinische Pharmakologie der Universität Zürich, die sich mit diesem Problem bereits auseinandergesetzt hat).

\section{Literatur}

1 Gnädinger M, Mellinghoff HU, Kälin-Lang A. Parkinson's disease and the bones. Swiss Med Wkly. 2011;141:w13154

2 Ross AC, Manson JE, Abrams SA, et al. The 2011 report on dietary reference intakes for calcium and vitamin $\mathrm{D}$ from the Institute of Medicine: what clinicians need to know. J Clin Endocrinol Metab. 2011;96:53-8. 